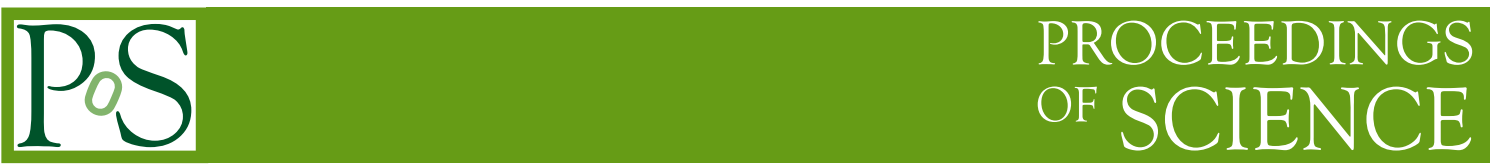

\title{
Calibration of the EUSO-TA Prototype Instrument
}

\author{
James Adams* \\ Univ. of Alabama in Huntsville \\ E-mail: jim.adams@uah.edu
}

Malek Mustafa, Matthew Rodencal, Evgeny Kuznetsov and John Watts

Univ. of Alabama in Huntsville

\section{Steven Csorna}

Vanderbilt University

\section{Lech Wiktor Piotrowski}

RIKEN

\section{for the JEM-EUSO Collaboration}

The Extreme Universe Space Observatory (EUSO) instrument is being developed for deployment on the International Space Station (ISS). Looking down from its berth on the ISS, EUSO will take high speed UV videos of Extensive Air Showers (EAS) caused by cosmic rays. Using these videos, the energy and arrival direction of each very high energy cosmic ray will be reconstructed. In order to reconstruct the energy, the absolute sensitivity of EUSO must be known. To test the EUSO instrument concept a prototype of EUSO (EUSO-TA) has been deployed on the Telescope Array (TA) site in Utah, USA. EUSO-TA takes advantage of the calibration facilities available on the TA site and the opportunity to observe extensive air showers recorded by the TA's fluorescence detectors. In this paper we describe the use of calibrated ultraviolet (UV) light emitting diodes (LEDs) to calibrate the EUSO-TA instrument.

The 34th International Cosmic Ray Conference,

30 July- 6 August, 2015

The Hague, The Netherlands

\footnotetext{
*Speaker.
} 


\section{Introduction}

A prototype of the Extreme Universe Space Observatory (EUSO) has been constructed to test the EUSO instrument concept. It is located on the Black Rock Mesa, part of the Telescope Array (TA) project near Delta, Utah. EUSO-TA is described in Adams et al. (2015) and Casolino et al. (2015).

A critical requirement for EUSO is that it must measure the absolute UV luminosity of extensive air showers (EASs) in the 330 to $400 \mathrm{~nm}$ range. Such measurements are needed to reconstruct the energy of the cosmic rays that initiate the EASs. In this paper we will describe how EUSO-TA was calibrated in order to correct for non-uniformities in the sensitivity of its focal surface and to determine its absolute sensitivity.

\section{The UV LED light sources}

In the work described here we used a $375 \mathrm{~nm}$ LED. It was used for the measurements to correct for the non-uniformities of the photon detection module (PDM) which serves as the focal surface in EUSO-TA. It was also absolutely calibrated against a reference phototube (Hamamatsu R8900-03) just before being used for calibrating the absolute sensitivity of EUSO-TA.

The LED source is shown in figure 1. The LED is mounted on the end of a small circuit board that provides its supporting electronics. In the final assembly, this circuit board is glued into the LED Driver Enclosure. A neutral density filter may be added in front of the LED and held on by the Neutral Density Enclosure which screws on the LED Driver Enclosure. The entire LED assembly fits into the Diffuser Housing. An opal diffuser may be installed in front of the LED assembly to produce a more uniform angular distribution of the light emitted by the LED.

The LED source was equipped with a collar permitting it to be fastened into the hole in the middle of the front lens of EUSO-TA as shown in figure 2. From this position it shone directly on

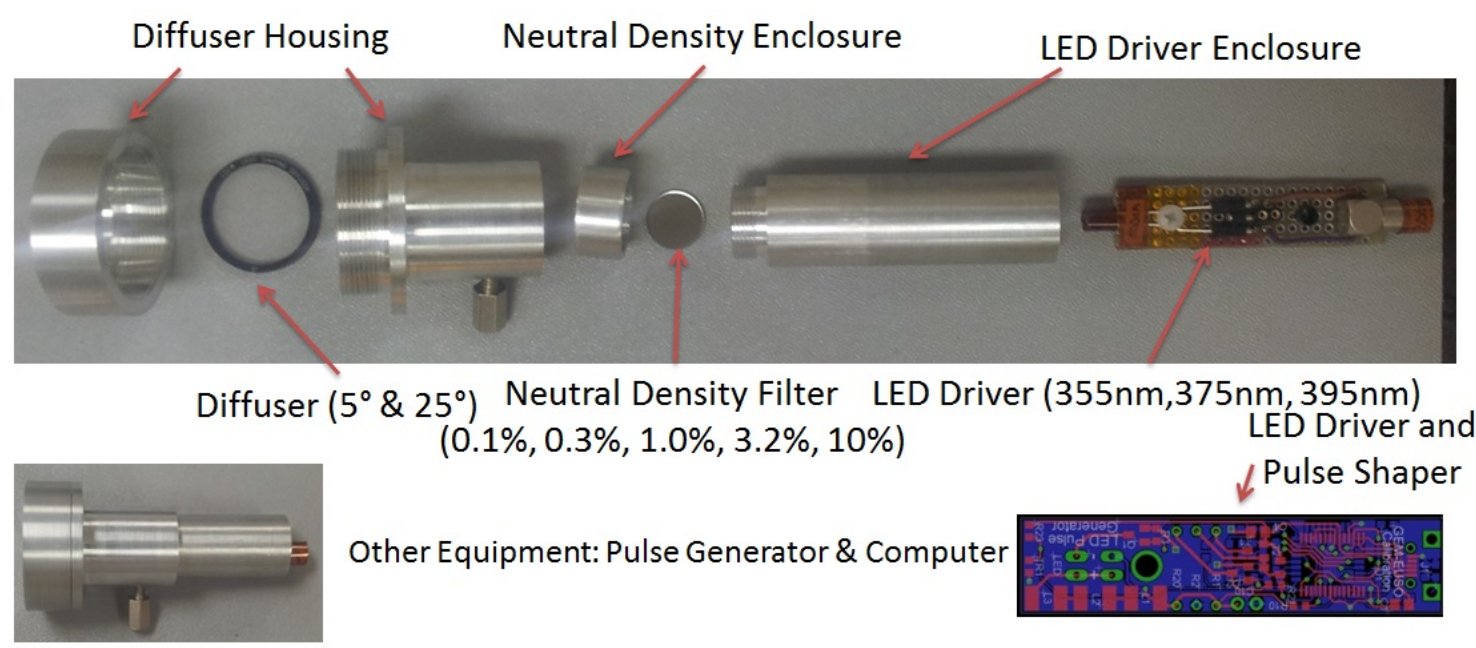

Figure 1: The LED source used for the measurements discussed in this paper. The details of the source are shown in the upper figure. The supporting circuit board is shown below on the right and the assembled source is shown on the lower left. 


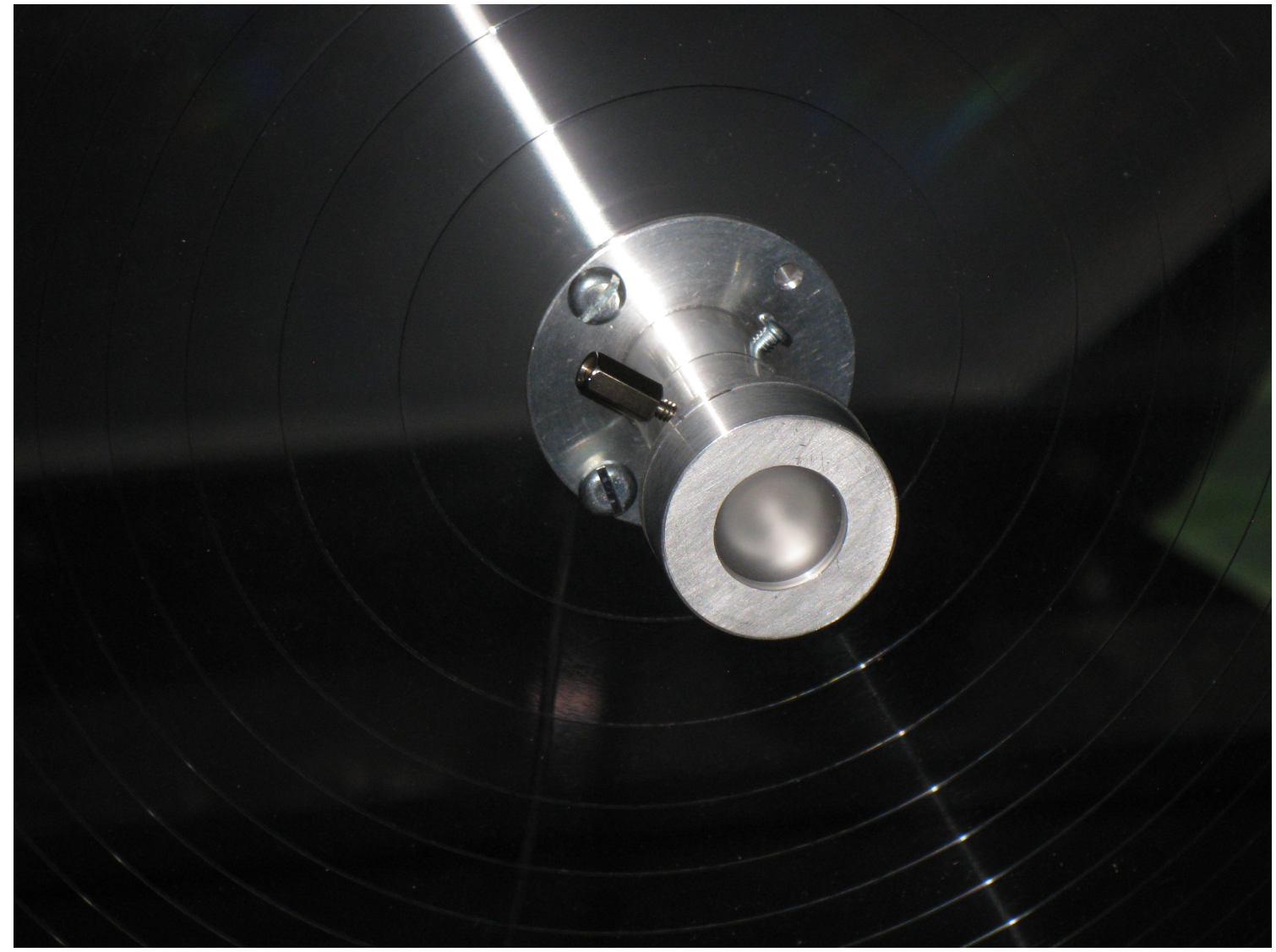

Figure 2: The LED Source mounted in the center of the front lens of EUSO-TA. From this position the LED was aimed at the center of the Flat Field Wall.

the Flat Field Wall (discussed in the next section) and was used to illuminate it. The light striking the viewed area of the wall was emitted from the LED at field angles up to $14.4^{\circ}$. The intensity of the light emitted by the LED depends on the angle of the emission (both field and azimuth angles). To improve the uniformity of the illumination of the wall $25^{\circ}$ opal diffuser was used. Even this was not enough to get completely uniform illumination.

The uniformity of the LED source using a $25^{\circ}$ opal diffuser was measured. To map out the intensity, measurements of the intensity were made at field angles of $0^{\circ}, 5^{\circ}, 10^{\circ}$ and $15^{\circ}$ and at azimuth angles in $45^{\circ}$ steps from $0^{\circ}$ to $360^{\circ}$. The results, fitted with a smooth surface, are shown in figure 3. As can be readily seen in this figure, the brightest illumination was not aimed at the center of the wall. This is because the LED is soldered onto the small circuit board and it was not possible to get it aimed perfectly. The measured intensity (in arbitrary units) was between 1.84 and 3.69 .

For an absolute calibration of EUSO-TA, this same LED source was placed on a table at a distance of 43.3 meters in front of EUSO-TA. The table was positioned so that the light from the LED would focus on one multi-anode photomultiplier tube (MAPMT) near its center so that the entire focal spot was captured by this MAPMT. Care was taken to aim the LED at the center of EUSO-TA's entrance aperture. In addition, masks were installed on the edge of the table holding 


\section{Illumination Pattern from $355 \mathrm{~nm}$ LED}

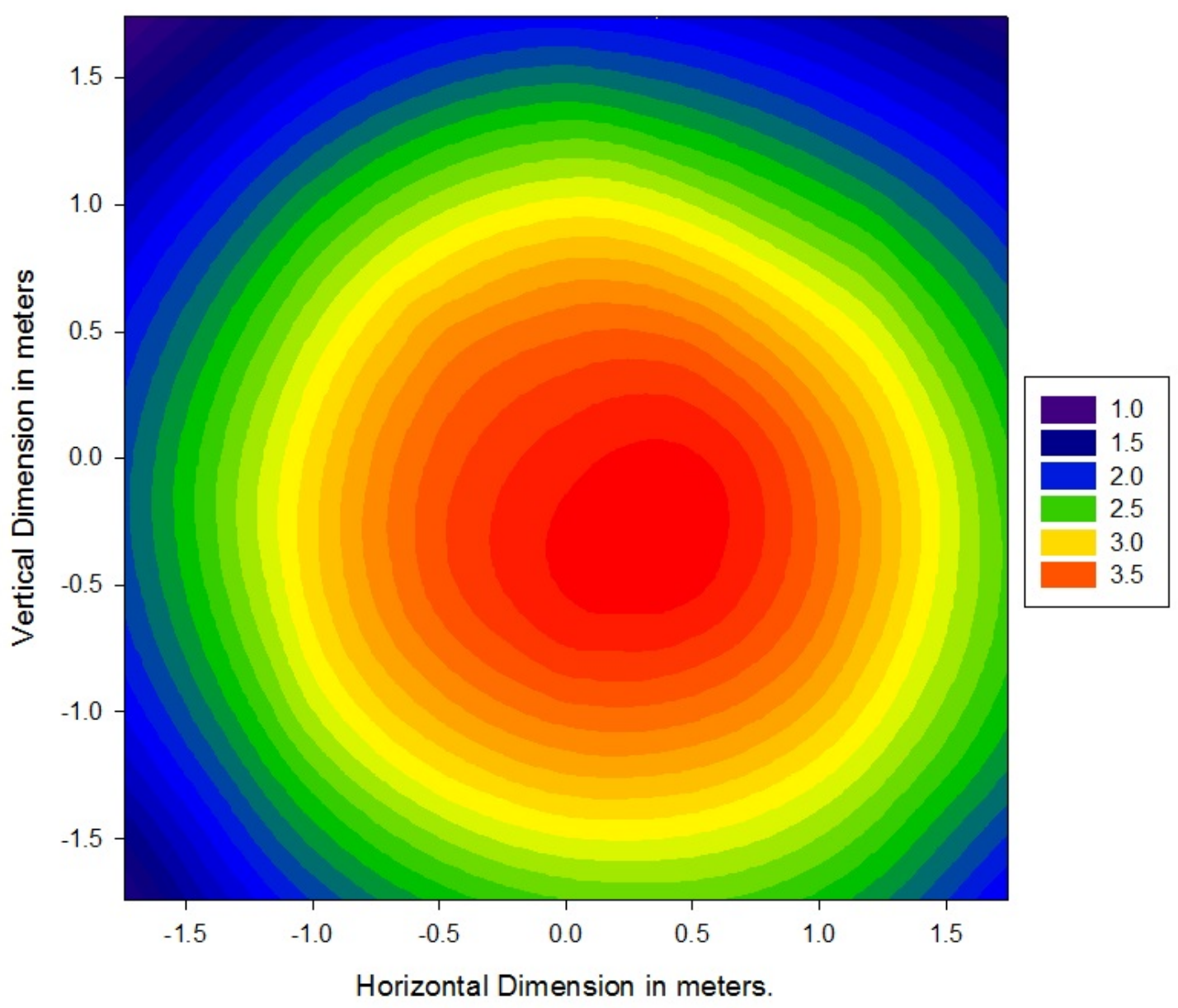

Figure 3: A fit to the intensity (in arbitrary units) of the light emitted by the $375 \mathrm{~nm}$ LED Source as a function of position on the Flat Field Wall. As can be seen, the LED inside the LED source was not perfectly pointed along the axis of the source. It should be noted that the Wall is only 2.44 meters on a side so the figure extends somewhat beyond the dimensions of the wall.

the LED and on another table placed in front of the LED to block any reflected light from reaching the entrance aperture. The LED was operated in pulsed mode. The angular non-uniformity of the LED was not important for this test because the maximum field angle was $0.93^{\circ}$. In addition, the reference phototube was positioned to subtend exactly the same solid angle as the entrance aperture of EUSO-TA so any effects from non-uniform illumination were calibrated out.

\section{Flat Field Wall}

In order to measure the non-uniformity in the response of the PDM, we constructed a wall that held a Tyvek screen. This screen was 2.44 by 2.44 meters and more than large enough to cover 


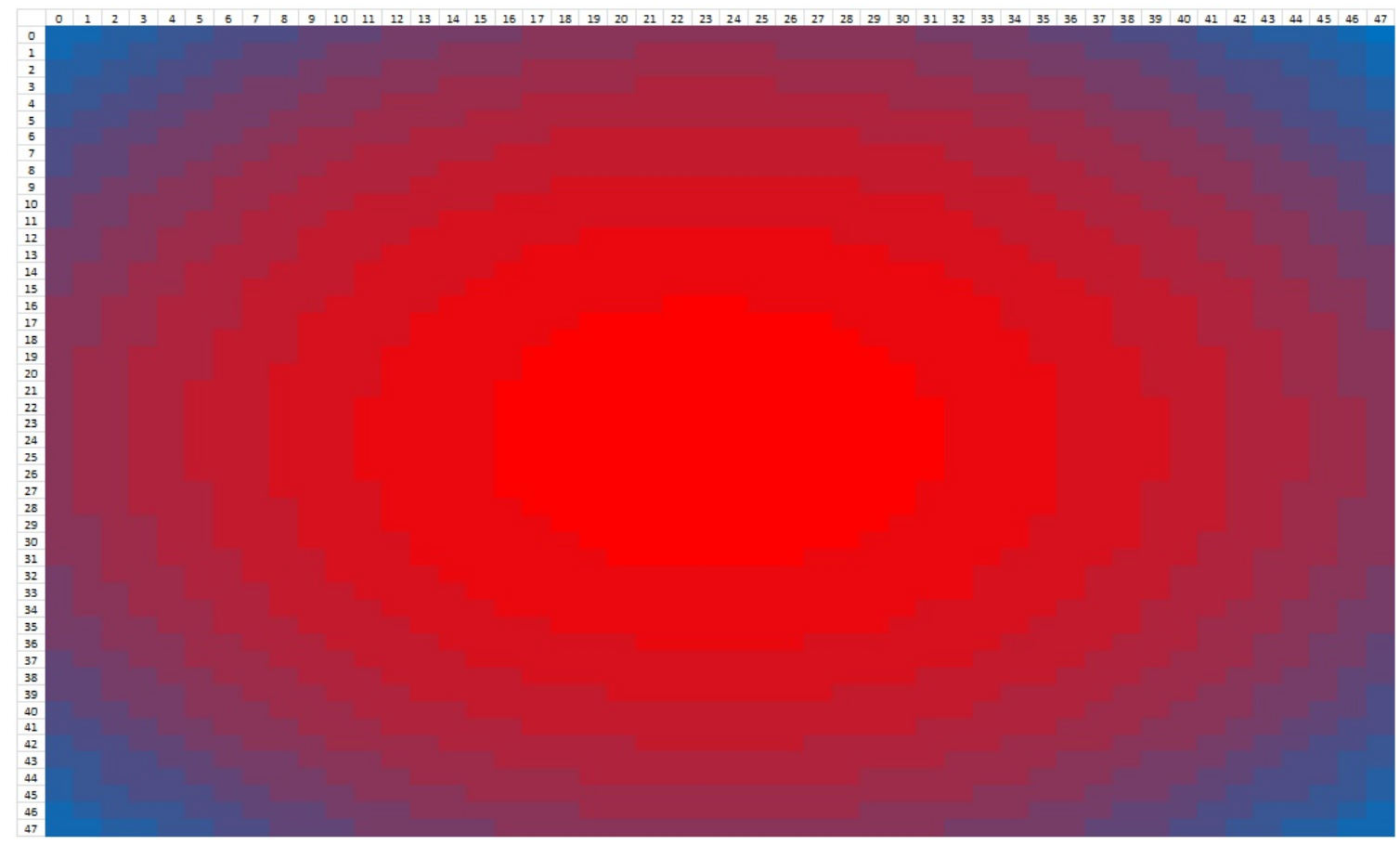

Figure 4: The 48 by 48 pixel map of the reflected light from the Flat Field Wall averaged over the area viewed by each pixel on the focal surface and normalized to 1.0. The values in the map range from 0.91 (blue) to 1.04 (red)

the entrance aperture of EUSO-TA. The screen was constructed from wood and is similar in design to an interior wall of a house. The wooden frame of the screen was covered with plywood. We stretched two layers of Dupont Tyvek over the screen to create a highly diffuse reflecting surface.

The wall was placed 6.5 meters in front of EUSO-TA and centered. This required EUSO-TA to be placed at an elevation angle of $7^{\circ}$ and the wall to be tilted toward EUSO-TA at the same angle. With these adjustments, the optical axis of EUSO-TA was aimed at the center of the screen.

The wall was illuminated by the $375 \mathrm{~nm}$ LED which was installed in the hole in the center of the front lens of EUSO-TA, pointing at the center of the wall. It must be noted that EUSO-TA was focused on infinity. For this reason, the light falling on any one point on the PDM came from an area on the wall of $1 \mathrm{~m}^{2}$ (or a little more at large field angles). This has the effect of smoothing out the non-uniform illumination from the LED source. To account for this in the analysis, we have averaged the reflected light over the area of the wall from which the light was collected and focused onto each pixel. This is an area surrounding the point where the principal ray for each pixel intersects the screen. The resulting map showing the average reflected light going to each pixel was normalized to 1.0 and is presented in figure 4. This map is used in the final analysis.

The reflectivity of Tyvek has been measured and carefully modeled (see Nozka et al., 2011). Using their results we have concluded that the departure from diffuse reflectivity of Tyvek is small and can be neglected in this investigation. 


\section{Results}

The PDM consists of an array of 6 by 6 MAPMTs. The borders around each photocathode and the gaps between the tubes are not sensitive. The PDM installed in EUSO-TA for the May, 2015 run had most of its MAPMTs working or partially working. In this paper we discuss calibrating those pixels in the PDM that were working properly.

We have obtained two results, the correction factors for each working pixel in the PDM that adjusts the sensitivity of each to the average sensitivity of the PDM. Second, we measured the sensitivity in one focal spot on one MAPMT. Applying the correction factors to the PDM, this one measurement becomes a measurement of the sensitivity of every pixel in the PDM.

\subsection{Flat Field Measurements}

The Flat Field measurements were made using the wall as described above. The LED was pulsed to provide both illuminated and background measurements in the same data file. The intensity of the LED was adjusted so that the photoelectron count per GTU was not high enough to cause pileup problems in the electronics (see Dagoret et al. (2015) for a discussion of pileup in the PDM electronics). We averaged the 'on' signal and the 'off' signal over a large number of GTUs to reduce the statistical error to a negligible level. We subtracted the 'off' signal from the 'on' signal to obtain a background-corrected measurement of the signal from the wall over the entire PDM.

In addition to the correction mentioned in the previous section, there is a geometrical correction to consider. The intensity of the LED source falls off as the inverse square of distance and it is farther to the corners of the wall than to the center. As mentioned above, the maximum field angle for the LED (i.e. for shining on the far corner of the $\sim 1 \mathrm{~m}^{2}$ viewed by a pixel in the corner of the $\mathrm{PDM}$ ) is $14.4^{\circ}$. The increased distance to this corner results in a $6.2 \%$ reduction in the light from the LED that reaches this corner. This small decrease in intensity is partly washed out because the light focused on the PDM is collected from $\sim 1 \mathrm{~m}^{2}$ area on the screen. Because of these considerations we have decided to neglect this correction in our analysis. So of the three possible corrections to the flat field measurement we will apply only the correction for the angular dependence of the intensity of the LED source. The results shown in figure 4 were used to correct the backgroundcorrected measurement of the signal from the wall in order to obtain the signal that the PDM would have measured had the wall been uniformly illuminated and diffusely reflecting. Finally, this result was normalized to 1 photoelectron per pixel per GTU in order to obtain the correction factors we are seeking. These factors are shown in figure 5.

\subsection{Calibration LED Measurements}

The calibration measurements were made using the arrangement described in section 2. The pulsed LED measurements were recorded at several LED intensities to find a signal level that was above background but not strong enough to cause saturation. The best signal level was obtained by using a $3.2^{\circ}$ neutral density filter and operating the LED at 3.12 volts. The resulting data set was analyzed, separating the LED 'on' and 'off' signals. Enough statistics were accumulated both with the LED 'on' and 'off' to make the statistical errors on the average 'on' and 'off' signals negligibly small. The 'off' average signal was then subtracted from the 'on' average to obtain the net signal. This signal was corrected using the matrix of correction factors shown in figure 5 to obtain the 


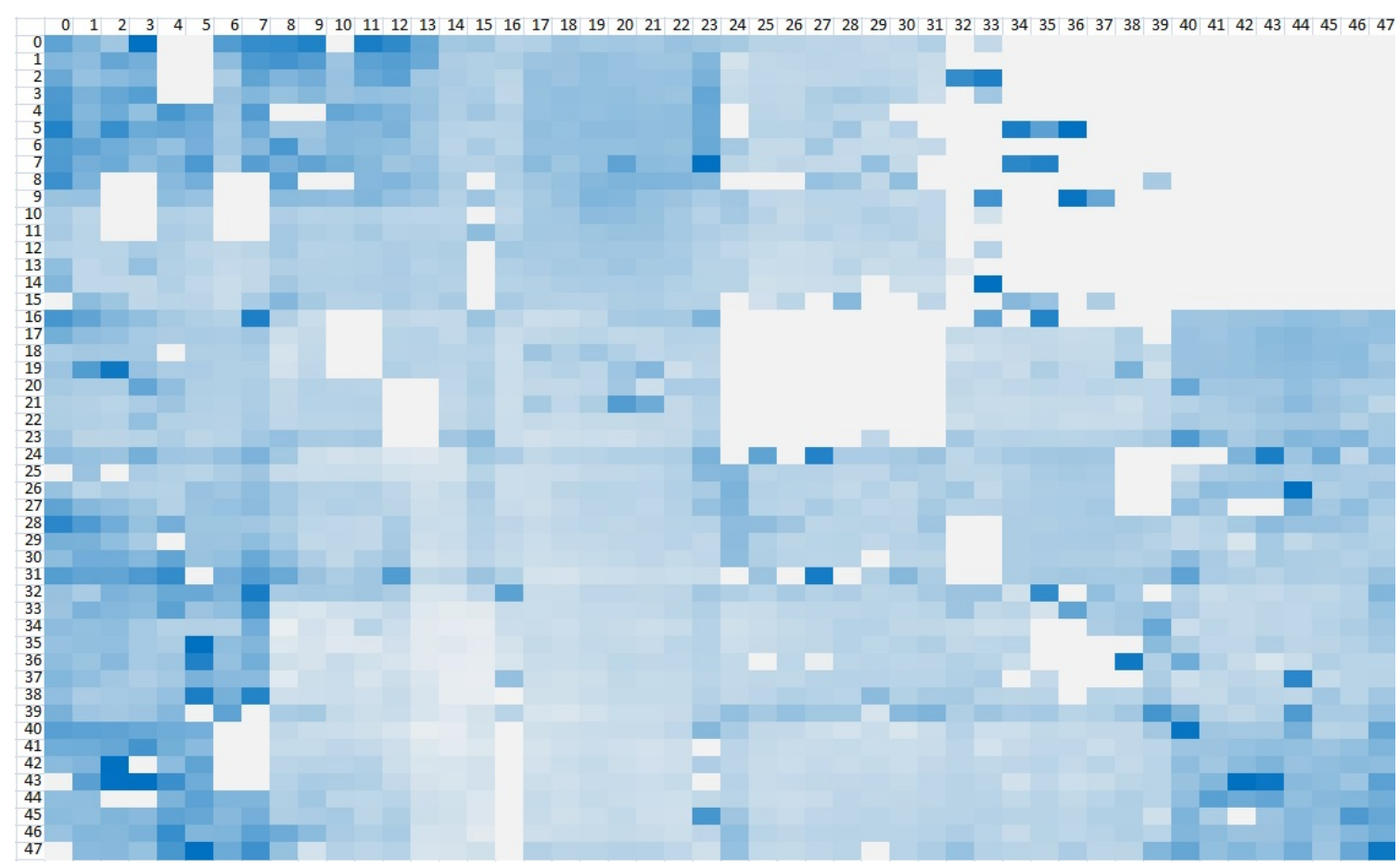

Figure 5: This figure shows a 48 by 48 pixel array of PDM correction factors for flattening the response. The scale is 0.0 (white) to 2.0 (dark blue). The white pixels are insensitive to light. The dark blue ones are the most sensitive. The scale is normalized so that average sensitive pixel has value of 1.0

corrected focal spot map shown in figure 6 . The integrated signal over a 5 by 5 pixel area centered on the focal spot yields 33 photoelectrons/GTU.

The $375 \mathrm{~nm}$ LED was calibrated the afternoon before these measurements were made with the neutral density filter and the voltage used. This calibration showed that the photon flux on the EUSO-TA entrance aperture should have been 2596 photons/GTU. Using nominal values for transmission of the optics, the quantum efficiency and the average sensitivity after the flat-field correction, this much light should have produced a signal of $\sim 111$ p.e.s/GTU but the signal was only 33 p.e.s/GTU. This difference may have been caused by the heavy dew that had settled on everything outdoors on Black Rock Mesa by 4:30 AM on the morning of May 23, 2015 when the measurements were made. The condensation on the LED could have caused a large reduction in the light reaching the entrance aperture of EUSO-TA. For this reason we regard our calibration as unreliable.

\section{Conclusions}

We have derived a matrix of correction factors to "Flat Field" the PDM used in EUSO-TA during the run of May, 2015. In addition, we have developed a procedure for determining the calibration factor for converting"Flat Field"-corrected photoelectron counts into photons incident on the entrance aperture of EUSO-TA. The calibration equipment and procedures that we have developed in the course of this work failed to work in this instance because of water condensation on the LED. Nevertheless we think this procedure is sound and can be applied to other PDMs and 


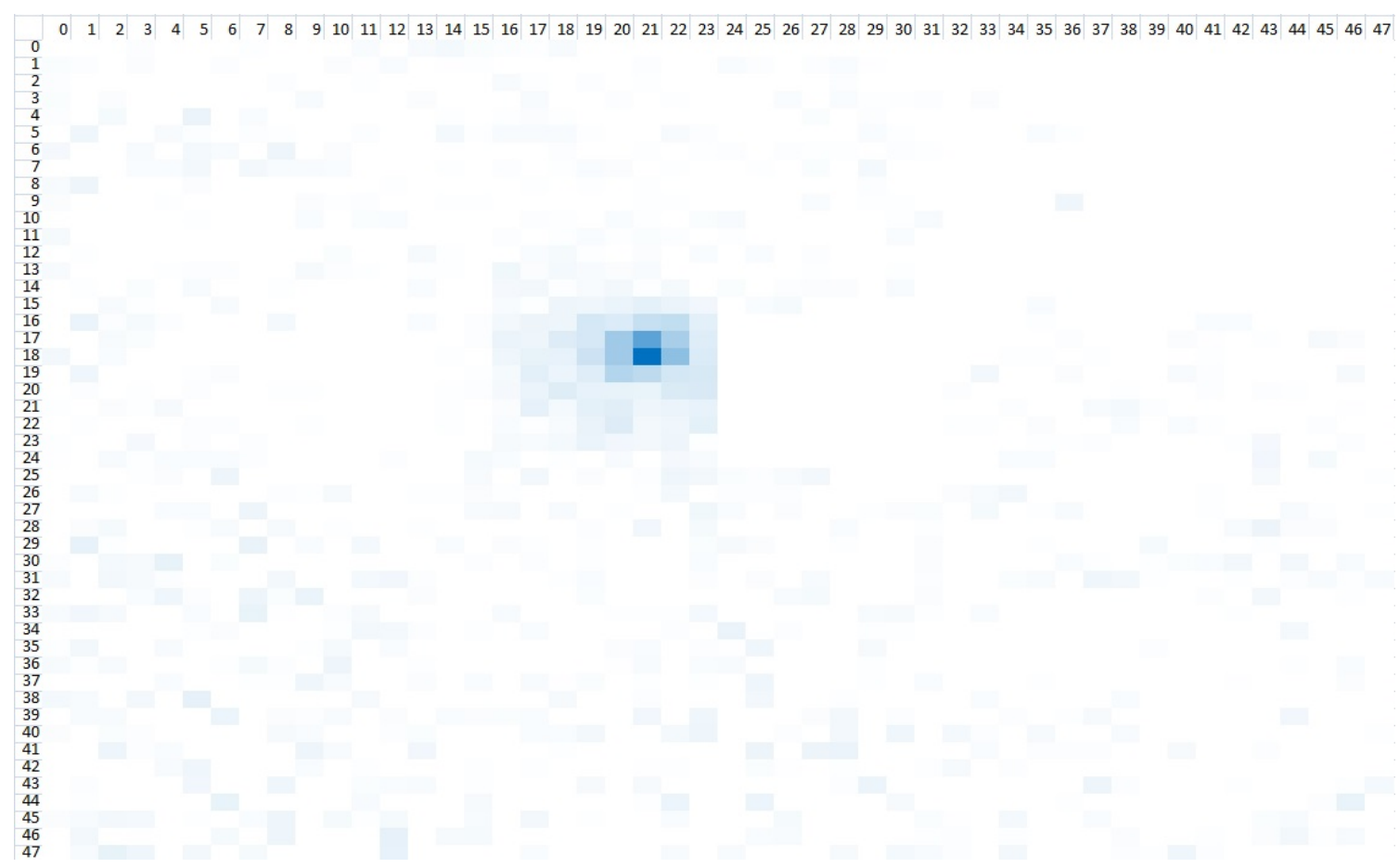

Figure 6: The calibration focal spot on the flattened 48 by 48 pixel PDM array. The scale is from 0 (white) to 7 (blue) photons/pixel. A 5 by 5 pixel array centered on this focal spot contains 33 photoelectons.

also to the calibration of EUSO Balloon prior to its super pressure balloon flight in the southern hemisphere in 2017.

Acknowledgment:The corresponding authors wish to thank Francesco Fenu and Zbigniew Plebaniak for operating EUSO-TA to collect the data for this paper and for providing some of the analysis software we used. The authors also wish to thank the Telescope Array Collaboration for hosting the support they provided during or work on Black Rock Mesa. This work was supported by NASA Grant NNX 13AH53G

\section{References}

[1] J.H. Adams, Jr. et al., "Ground-based tests of JEM-EUSO components at the Telescope Array site, 'EUSO-TA"', Experimental Astronomy, DOI:10.1007/s10686-015-9441-6 (2015).

[2] M. Casolino et al., "Detecting ultra-high energy cosmic rays from space with unprecedented acceptance: objectives and design of the JEM-EUSO mission collaboration”, Astrophys. Space Sci. Trans., Vol. 7, 477-482 (2011).

[3] S. Dagoret et al., "The Photodetector Module of the JEM-EUSO mission", Experimental Astronomy (in press) (2015).

[4] Libor Nozka el al., "BRDF profile of Tyvek and its implementation in the Geant4 simulation toolkit", Optics Express, Vol. 19, No. 5 (2011). 